\title{
A Motion Analysis of the Bunraku Puppet based on Linear Structure of Functional Factors, Emotional Factors and Stochastic Fluctuations \\ - For generation of home robots' actions with fertile emotions -
}

\author{
M.Hattori, M.Tsuji, S.Tadokoro and T.Takamori \\ Kobe University, Fac. of Eng., Dept. of Computer and Systems Eng. \\ (Rokkodai-cho, Nada-ku, Kobe 657 Japan) \\ Tel. +81-78-803-1200, Fax. +81-78-803-1217 \\ E-mail: hattori@in.kobe-u.ac.jp, UR.J : http://robomec.cs.kobe-u.ac.jp
}

\begin{abstract}
Human robots must have actions with fertile emotions, in order to cooperate with human being. To clarify "what is the emotional factor of actions", the authors analyze the actions of a Bunraku puppet. The observed action time serics are modeled by a stochastic time evolution equations $\left(\mathrm{KM}_{2} \mathrm{O}\right.$-Langevin equations) with exogenous sources which represent the sources of functional factors, emotional factors and stochastic fluctuation factors. The estimation method for $\mathrm{KM}_{2} \mathrm{O}$-Langevin equations with exogenous sources is established. This method is verified by a numerical simulation.
\end{abstract}

Keywords : Home robot, Human-like action, Emotion, Feeling, Bunraku, $\mathrm{KM}_{2} \mathrm{O}$-Langevin equation

\section{Introduction}

Robots have played mainly important roles in factories up to the present and will be active in our daily life in future in our home[1]. In order to cooperate with human being, it is desirable that these robots act with fertile emotions. To grasp the essence of the emotional actions, the authors pay attention to the actions of Bumraku puppets.
Bunraku is a classical entertaimment in Japan. It is a puppet show in which the story is narrated and sung to a 'shamisen' (a Japanese stringed instrument) accompaniment. In this show, the typical emotional actions are generated by experts of puppet manipulation.

In this study, the motions of a Bunraku puppet are measured and analyzed. We measure the actions of plural functions and plural emotions which are generat,ed by experts of puppet manipulation. These action time series are analyzed based on the hypothesis that the time series is consist of a functional factor, an emotional factor and a stochastic fluctuation factor. If the stochastic fluctuation factors are weakly stationaly stochastic processes, the action time series are expressed by Kubo,Mori,Miyoshi,Okabe's Langevin equations ( $\mathrm{KM}_{2} \mathrm{O}$-Langevin equations) $[3][4]$ with exogenous sources which represent the source of functional factors, emotional factors and stochastic fluctuation factors.

The method to estimate these sources are established in this paper. Based on the estimated sources, the functional factors, the emotional factors and the stochastic fluctuation factors are computed. These knowleclge will be applied to generate actions of home robots with fertile emotions. 


\section{Experimental setup}

In order to measure the actions, a woman puppet is made for the experimental use. A Bumraku puppet is constructed with a head, a shoulder board, a waist ring and two arms. A man puppet has legs, but a woman puppet does not, have legs.

$\Lambda$ puppet. is manipulated by three puppet manipulators; one manipulates her head and right arm, the another manipulates left arm and the remainder legs.

In orcler to measure the positions and the postures of the puppet, the tracking system FASTRAK is used. FASTRAK generates magnetic field vectors from a single assembly of three collocated antemnas called a 'transmitter' and detect the field vectors with a single assembly of three collocated, remote sensing antemnas called a 'receiver'.

The four receivers are installed on the parts of the puppet as shown. The 1 st $\operatorname{sensor}(p=1)$ is installed on the head of the puppet, the 2 nd $\operatorname{sensor}(p=2)$ is installed on the shoulder, the $3 \mathrm{rd} \operatorname{sensor}(p=3)$ is installed on the right arm and the 4 th sensor $(p=4)$ is installed on the right, hand.

We have $M=4$ receivers(sensors). Each sensor observes position (3 degree of freedom) and posture (3 degree of freedom). The time series of clata observed by $p$-th sensor is described as

$$
x_{p}(t)=\left[x_{p q}(t) ; q \downarrow 1, \cdots, 6\right]
$$

where $x_{p 1}(t)$ is the $x$ coordinate, $x_{p 2}(t)$ is the $y$ coordinate, $x_{p 3}(t)$ is the $z$ coordinate, $x_{p 4}(l)$ is the yaw $\psi, x_{p 5}(t)$ is the pitch $\theta$ and $x_{p 6}(t)$ is the roll $\phi$.

Then the state $x(t)$ of the puppet can be described as

$$
x(t)=\left[x_{p}(t) ; p \downarrow 1, \cdots, M\right]
$$

In our experiment, we have 4 sensors, i.e. $M=4$. For one action $\left\{x(t) ; t=0,1,2, \cdots, T_{f}\right\}$, the dimension of the state $x(t)$ is $6 M=24$. The sampling time of our experiment was $0.2[s \in c]$.
Table 1: Functional and emotional factors

\begin{tabular}{|l|l|}
\hline functional factors & emotional factors \\
\hline \hline$i=1:$ greeting & $j=1:$ no emotion \\
\hline$i=2:$ serving tea & $j=2:$ friendly emotion \\
\hline & $j=3:$ hostile emotion \\
\hline
\end{tabular}

\section{Observed action time series}

In order to see the characteristics of actions depending on their functional factors and emotional factors, we measure action time series $x^{i j}(t)(t=$ $0,1,2, \cdots, T_{f}$ ) for plural functions $i$ and plural emotions $j$. In this experiment, the considered functional factors are to greet $(i=1)$ and to serve tea $(i=2)$, and the considered emotional factors are friendly feeling $(j=2)$, $\operatorname{los}$ tile feeling $(j=3)$ and no emotion $(j=1)$. (See Table 1.)

In order to extract the stochastic fluctuation in actions, action $x^{i j}(t)\left(t=0,1,2, \cdots, T_{f}\right)$ are repeated $K$ times under same function $i$ and same emotion $j$. Thus we measured time series $x_{k}^{i j}(t)$ $\left(t=0,1,2, \cdots, T_{f}\right)(i=1, \cdots, I, j=1, \cdots, J$, $k:=1,2, \cdots, K)$, where $I=2, J=3$ and $K=5$ in our experiment.

\section{Functional factors, emo- tional factors and stochastic factors of action time series}

In this paper, these action time series are analyzed based on the following hypotheses. The validity of these hypotheses must be checked based on the real action time series.

\section{Hypothesis 1}

The action time series is decomposed into a functional factor, an emotional factor, and a stochastic fluctuation factor in time domain as follows:

$$
x_{k}^{i j}(t)=y^{i}(t)+z^{i j}(t)+\eta_{k}^{i j}(t)
$$

- $y^{i}(t)$ : functional factor

- $z^{i j}(t)$ : enotional factor 
- $\eta_{k}^{i j}(t)$ : stochastic fluctuation

\section{Hypothesis 2}

- functional factor $y^{i}(t)$ : deterministic

- emotional factor $z^{i j}(t)$ : deterministic

For $j=1$ (no emotion), the emotional factors are zero, that is,

$$
z^{i 1}=0 \quad(i=1,2)
$$

Hypothesis 3

$\left\{\eta_{k}^{i j}(t) ; t=0,1,2, \cdots, T_{f}\right\}$ is a weakly stationary stochastic process and $\left\{\eta_{k}^{i j}(\cdot)\right\}_{k=1,2, \cdots, K}$ are independent and identically distributed.

\section{Representation of action} time series based on $\mathrm{KM}_{2} \mathrm{O}$ Langevin equations with exogenous sources

Since $\left\{\eta_{k(p q)}^{i j}(t) ; t=0,1,2, \cdots, T_{f}\right\}$ is a weakly stationary stochastic process, it is represented by the following

Kubo,Mori,Miyoshi,Okabe's Langevin equation ( $\mathrm{KM}_{2} \mathrm{O}$-Langevin equation) [3][4]

$$
\begin{aligned}
\eta_{k(p q)}^{i j}(t)= & -\sum_{s=0}^{t-1} \gamma_{+(p q)}^{i j}(t, s) \eta_{k(p q)}^{i j}(s) \\
& +\nu_{+k(p q)}^{i j}(t)
\end{aligned}
$$

where $\gamma_{+(p q)}^{i j}(t, s)$ is a deterministic scalor and $\nu_{* k(p q)}^{i j}(\cdot)$ is a white noise whose probability distribution is not necessarily Gaussian. The variance of the white noise $\nu_{+k(p q)}^{i j}(t)$ is denoted by $V_{+(p q)}^{i j}(t)$.

$\mathrm{KM}_{2} \mathrm{O}$-Langevin equation call represent every weakly stationary stochastic process even though it does not satisfy

- finitely Markovian property

- Gaussian probability distribution
Since the stochsatic fluctuation factor $\eta_{k}^{i j}(t)$ is expressed by $\mathrm{KM}_{2} \mathrm{O}$-Langevin equation, the action time series $\left\{x_{k}^{i j}(t) ; t=0,1,2, \cdots, T_{f}\right\}$ satisfies $\mathrm{KM}_{2} \mathrm{O}$-Langevin equation with exogenous sources

$$
\begin{aligned}
x_{k(p q)}^{i j}(t)= & -\sum_{s=0}^{t-1} \gamma_{+(p q)}^{i j}(t, s) x_{k(p q)}^{i j}(s) \\
& +u_{(p q)}^{i}(t)+v_{(p q)}^{j}(t)+v_{+k(p q)}^{i j}(t)
\end{aligned}
$$

where functional sources $u^{i}(t)$ and emotional sources $v^{i j}(t)$ are clefined as follows:

Definition

$$
\begin{aligned}
& u_{(p q)}^{i}(t)=y_{(p q)}^{i}(t)+\sum_{s=0}^{t-1} \gamma_{+(p q)}^{i j}(t, s) y_{(p q)}^{i}(s) \\
& v_{(p q)}^{i j}(t)=z_{(p q)}^{i j}(t)+\sum_{s=0}^{t-1} \gamma_{+\cdot(p q)}^{i j}(t, s) z_{(p q)}^{i j}(s)
\end{aligned}
$$

In later description, the subscript $(p, q)$ is omitted for simplicity.

$$
\begin{aligned}
x_{k}^{i j}(t)= & -\sum_{s=0}^{t-1} \gamma_{+}^{i j}(t, s) x_{k}^{i j}(s) \\
& +u^{i}(t)+v^{i j}(t)+\nu_{+k}^{i j}(t)
\end{aligned}
$$

$V_{+}^{i j}(t)$ is a variance of the white noise $\nu_{+k}^{i j}(t)$.

\section{Methods to estimate pa- rameters and sources of the $\mathrm{KM}_{2} \mathrm{O}$-Langevin equa- tions with exogenous sources}

In order to estimate the parameters $\gamma_{+}^{i j}(t, s)$ $\left(0 \leq s<t \leq T_{f}\right)$ and the variance $V_{+}^{i j}(t)$ $\left(t=0,1,2, \cdots, T_{f}\right)$ based on the observed time series (sample paths of the stochastic processes $\left.\left\{x_{k}^{i j}(t) ; t=0,1,2, \cdots, T_{f}\right\}\right) \quad(i=1, \cdots, I, \quad j=$ $1, \cdots, J, k=1,2, \cdots, K)$, we derive the stationary stochastic process $\left\{x_{(\text {diff }) k}^{i j}(t) ; t=0,1,2, \cdots, T_{f}\right\}$ by eliminating the exogenous sources $u^{i}(t)$ and $v^{i j}(t)$ from the non-stationary stochastic process $\left\{x_{k}^{i j}(t) ; t=0,1,2, \cdots, T_{f}\right\}$ as follows. 
Let $x_{(\text {mean })}^{i j}(t)$ be a sample average of $x_{k}^{i j}(t)(k=$ $1,2, \cdots, K)$, that is,

$$
x_{(\text {mean })}^{i, j}(t)=\frac{1}{K} \sum_{k=1}^{K} x_{k}^{i j j}(t)
$$

By eq. (8), we have

$$
\begin{aligned}
x_{\text {(mean) }}^{i j}(t)= & -\sum_{s=0}^{t-1} \gamma_{+}^{i j}(t, s) x_{(\text {mean })}^{i j}(s) \\
& +u^{i}(t)+v^{i j}(t) \\
& +\frac{1}{K} \sum_{k^{\prime}=1}^{K} \nu_{+k^{\prime}}^{i j}(t)
\end{aligned}
$$

The stationary stochastic process $x_{(\text {diff }) k}^{i j}(t)$ is defined as

$$
x_{(\text {diff }) k}^{i j}(t)=x_{k}^{i j}(t)-x_{(\text {mean })}^{i j}(t)
$$

and it satisfies

$$
\begin{aligned}
x_{(d i f f) k^{i j}}^{i j}(l)= & -\sum_{s=0}^{t-1} \gamma_{+}^{i j}(t, s) x_{\left(d i f \int\right) k^{i j}}^{i j}(s) \\
& +\nu_{+k}^{i j}(t)-\frac{1}{K} \sum_{k^{\prime}=1}^{K} \nu_{+k^{\prime}}^{i j}(t)
\end{aligned}
$$

Since $\left\{x_{(\text {diff }) k}^{i j}(t) ; t=0,1,2, \cdots, T_{f}\right\}$ is a stationary stochastic process, the parameter $\gamma_{+}^{i j}(t, s)$ in eq. (12) and the variance $V_{+(\text {diff })}^{i j}(t)$ of $\nu_{+k}^{i j}(t)-$ $\frac{1}{K} \sum_{k^{\prime}=1}^{K} \nu_{+k^{\prime}}^{i j}(t)$ are estimated from the sample autocovariance function

$$
\begin{aligned}
& R_{(\text {diff }) k}^{i j}(\tau)= \\
& \frac{1}{1+T_{f}} \sum_{s=0}^{T_{f}-\tau} x_{(\text {diff }) k}^{i j}(s+\tau) x_{(\text {diff }) k}^{i j}{ }^{T}(s)
\end{aligned}
$$

of $\left\{x_{(d i f f) k}^{i j}(t) ; t=0,1,2, \cdots, T_{f}\right\}$ by the fluctuation dissipation theorem proposed by Y.Okabe [3][4].

The variance $V_{+}^{i, j}(t)$ of the stochastic fluctuation force $\nu_{+k}^{i j}(t)$ is computed from the variance $V_{+(d i f f)}^{i j}(t)$ of $\nu_{+k}^{i \cdot j}(l)-\frac{1}{K} \sum_{k^{\prime}=1}^{K} \nu_{+k^{\prime}}^{i, j}(t)$, since

$$
\begin{aligned}
& V_{+(\text {diff })}^{i j}(t) \\
= & \operatorname{Var}\left[\nu_{+k}^{i j j}(t)-\frac{1}{K} \sum_{k^{\prime}=1}^{K} \nu_{+k^{\prime}}^{i j}(t)\right] \\
= & \operatorname{Var}\left[\left(1-\frac{1}{K}\right) \nu_{+k}^{i j}(t)+\frac{1}{K} \sum_{k^{\prime} \neq k^{\prime}} \nu_{+k^{\prime}}^{i j}(t)\right]
\end{aligned}
$$

$$
\begin{aligned}
& =\left(1-\frac{1}{K}\right)^{2} V_{+}^{i j}(t)+\frac{K-1}{K^{2}} V_{+}^{i j}(t) \\
& =\frac{K-1}{K} V_{+}^{i j}(t)
\end{aligned}
$$

The Markov estimates of functional source $u^{i}(t)$ and the emotional source $v^{i j}(t)$ are computed from the following linear simultaneous equations

$$
\begin{aligned}
& x_{(\text {mean })}^{i j}(t)+\sum_{s=0}^{t-1} \gamma_{+}^{i j}(t, s) x_{(\text {mean })}^{i j}(s) \\
= & u^{i}(t)+v^{i j}(t) \\
& +\frac{1}{K} \sum_{k^{\prime}=1}^{K} l_{+k^{\prime}}^{i j}(t)
\end{aligned}
$$

for $i=1,2, \cdots, I ; j=1,2, \cdots, J$

based on the estimate of the covariance matrix $V_{+}^{i j}(t)$ of stochastic fluctuation force $\nu_{+k}^{i j}(t)$ computed by the above equation (16) [2].

\section{Numerical simulation to ver- ify the proposed estimation method}

In order to verify the proposed algorithm which estimates the sources of the stochastic time evolution equations ( $\mathrm{KM}_{2} \mathrm{O}$-Langevin equations), the following numerical simulation was made.

Time series $\left\{x_{k}^{i j}(t) ; t=0,1, \cdots, T_{f}\right\}(i=1,2 ; j=$ $1,2,3 ; k=1,2, \cdots K ; K=5$ ) were generated as follows.

$$
\begin{aligned}
x_{k}^{11}(t)= & -\frac{1}{2} x_{k}^{11}(t-1)-\frac{1}{3} x_{k}^{11}(t-2) \\
& +2 \sin \left(\frac{10}{140} t\right)+c w_{k}^{11}(t) \\
x_{k}^{12}(t)= & -\frac{1}{2} x_{k}^{12}(t-1)-\frac{1}{3} x_{k}^{12}(t-2) \\
& +2 \sin \left(\frac{10}{140} t\right)-2 \cos \left(\frac{10}{120} t\right)+c w_{k}^{12}(t) \\
x_{k}^{13}(t)= & -\frac{1}{2} x_{k}^{13}(t-1)-\frac{1}{3} x_{k}^{13}(t-2) \\
& +2 \sin \left(\frac{10}{140} t\right)-2 \cos \left(\frac{10}{100} t\right)+c w_{k}^{13}(t) \\
x_{k}^{21}(t)= & -\frac{1}{4} x_{k}^{21}(t-1)-\frac{1}{5} x_{k}^{21}(t-2) \\
& +3 \sin \left(\frac{10}{140} t\right)+c w_{k}^{21}(t)
\end{aligned}
$$




$$
\begin{aligned}
x_{k}^{22}(t)= & -\frac{1}{4} x_{k}^{22}(t-1)-\frac{1}{5} x_{k}^{22}(t-2) \\
& +3 \sin \left(\frac{10}{140} t\right)-3 \cos \left(\frac{10}{120} l\right)+c w_{k}^{22}(t) \\
x_{k}^{23}(t)= & -\frac{1}{4} x_{k}^{23}(t-1)-\frac{1}{5} x_{k}^{23}(t-2) \\
& +3 \sin \left(\frac{10}{140} t\right)-3 \cos \left(\frac{10}{100} t\right)+c w_{k}^{23}(t)
\end{aligned}
$$

where $w_{k}^{i j}(t)$ are white Gaussian noises of variances 1 , $k=1,2, \cdots, K ; K=5 ; \quad t=1,2, \cdots, T_{f} ; T_{f}=300 ;$ $c=1.4 \times 10^{-3}$, and $x(0)=10, x(1)=5$.

The sources $u^{i}(t), v^{i j}(t)(i=1,2 ; j=1,2,3)$ are estimated from the above time series $x_{k}^{i j}(t)$ based on the proposed algorithm. The true values and the estimates are shown in Figure $1,2, \cdots, 6$. These results show that the sources are estimated well by the proposed algorithm.

Since the sample auto-covariance function $R_{(d i f f) k}^{i j}(t)$ defined by eq.(13) is not effective if $t$ is large, the parameter $\gamma_{+}^{i j}(t, s)$ is not effoctive if $t$ is large. Indded $\left\{R_{\left(d i . j \int\right) k}^{i j}(t) ; t=0,1, \cdots, M_{f}\right\}$ and $\left\{\gamma_{+}^{i j}(t, s) ; 0 \leq s<t \leq M_{f}\right\}$ are effective where

$$
M_{f}=\left[3 \sqrt{T_{f}+1}\right]-1 .
$$

Since $\eta_{k}^{i j}(\cdot)$ is a weakly-stationary stochastic process, the parameter $\gamma_{+}^{i j}(t, s)$ does not depend on the initial time $T_{0}\left(T_{0}=0,1, \cdots, T_{f}-M_{f}\right)$ and we get, the following $\mathrm{KM}_{2} \mathrm{O}$-Latungevin equation

$$
\begin{aligned}
\eta_{k}^{i j}\left(T_{0}+t\right)= & \sum_{s=0}^{t-1} \gamma_{+}^{i j}(t, s) \eta_{k}^{i j}\left(T_{0}+s\right) \\
& +-\nu_{+k}^{i j}\left(T_{0}+t\right)
\end{aligned}
$$

with initial time $T_{0}$, according to [4][5].

Thus, in the above estimation (numerical simulation), we used $\mathrm{KM}_{2} \mathrm{O}$-Langevin equation

$$
\begin{aligned}
x_{k}^{i j}\left(T_{0}+t\right)= & -\sum_{s=0}^{t-1} \gamma_{+}^{i j}(t, s) x_{k}^{i j}\left(T_{0}+s\right) \\
& +u^{i}\left(T_{0}+t\right)+v^{i j}\left(T_{0}+t\right)+\nu_{+k}^{i j}\left(T_{0}+t\right)
\end{aligned}
$$

with initial time $T_{0}$.

\section{Conclusions}

The Bunraku puppet's action time series are expressed $\mathrm{KM}_{2} \mathrm{O}$-Langevin equations with exogenous sources which represent the source of functional fac1.ors, emotional factors and stochastic fluctuation facl.ors.

The method to estimate these sources are established. The numerical simulation verified the effectiveness of the proposed estimation method.

Acknowledgments : The authors would like t.o express their thanks to Prof. Kazuhito YAMADA (Doshisha. Univ, l'ace of Litcrature) for his valuable advices. They also express their thanks to Mr. Tamanne YOSHIDA for his kind puppet. manipulation. This research is supported by Hayao Nakayama foundation for science \& technology and culture. It is also supported by the grant-in-aid for scientific research on priority areas "intelligent robot." (No.09221218) and on encouragement researches (No.09750298) in Japan. These supports are much appreciated by the aluthors.

\section{References :}

[1] Special issue on new technologies for humanrobot cooperation - toward machina sapiens : new machine - Journal of the Society of Instrument, and (ontrol Engineers, vol.34, no.4, 1995 (in Japranese)

[2] T'Nakannizo, "Signal analysis and system identification", pp.10-13, Corona publishing co., 1988 (in Japanese)

[3] Y.Okabe, "Langevin equations and causal analysis", Amer. Math. Soc. Transl. (2) vol.161, pp. 19-50, 1994

[4] Y.( )kabe and Y.Nakano, "The theory of $\mathrm{KM}_{2} \mathrm{O}$ Langevin equations and its applications to data analysis (1) : Stationary analysis", Hokkaido Mathematical Joumal, vol.20, pp.45-90, 1991

[5] H.Kanvashima and 1I.Sakai, "Modern spectial analysis", pp.164, Norikita publishing co., 1989 (in Japanese) 


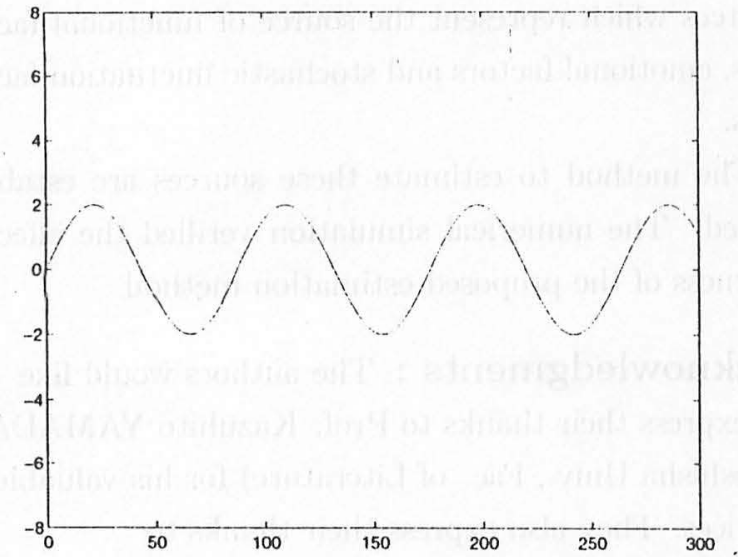

Fig. 1: The true value of $u^{1}(t)=2 \sin \left(\frac{10}{140} t\right)$

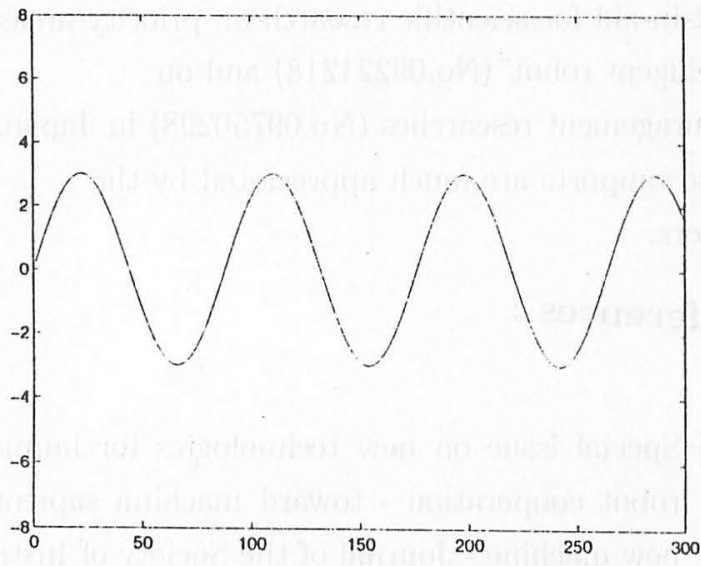

Fig. 2: The true value of $u^{2}(t)=3 \sin \left(\frac{10}{140} t\right)$

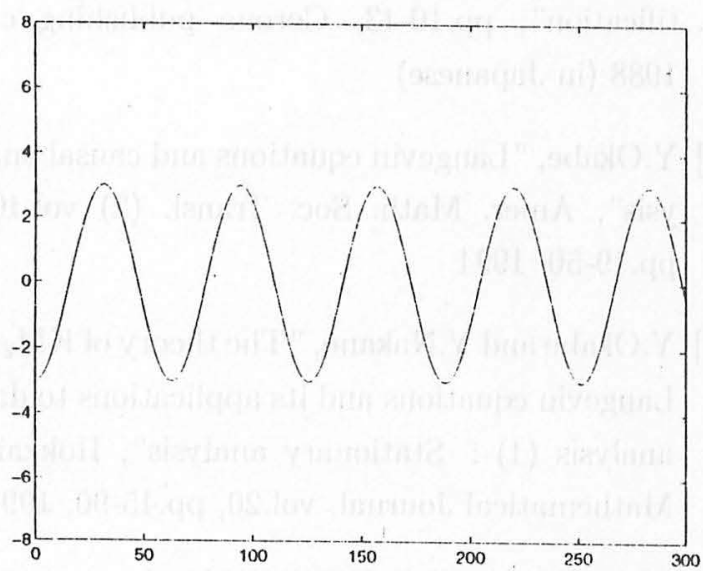

Fig. 3: The true value of $v^{23}(t)=-3 \cos \left(\frac{10}{100} t\right)$

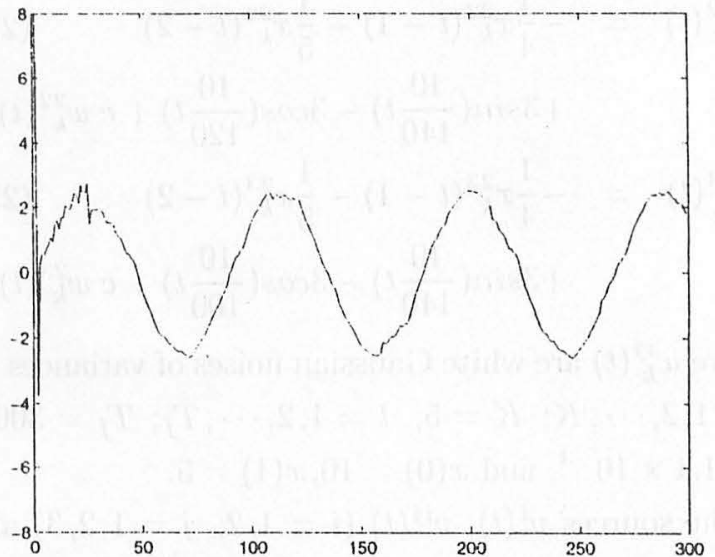

Fig. 4: The estimate of $u^{1}(t)$

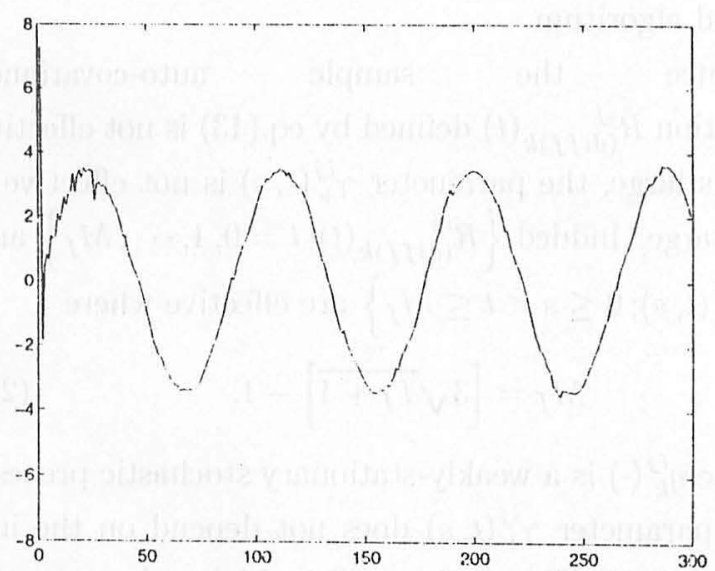

Fig. 5: 'The estimate of $u^{2}(t)$

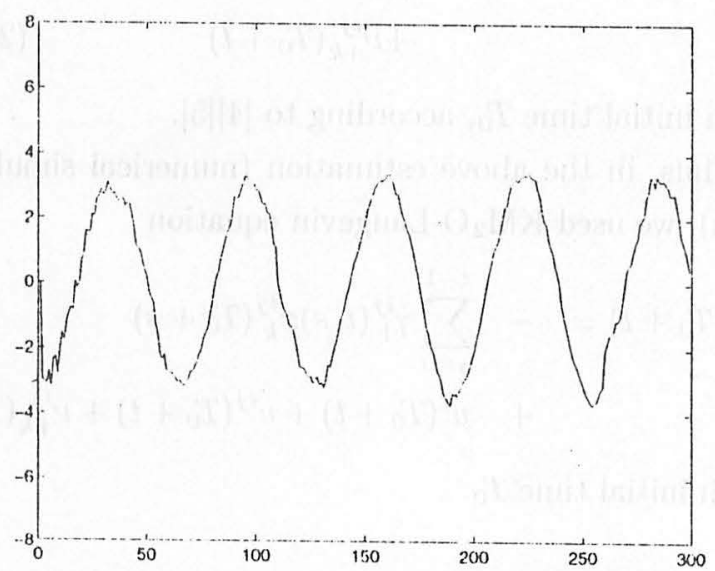

Fig. 6: The estimate of $v^{23}(t)$ 